\title{
Sylvestre Clancier, un compagnon inquiet
}

\author{
Bernard Fournier \\ Paris
}

$\underset{1}{ }$

isons ce poème liminaire de la dernière livraison de Poésie sur Seine qui par son début et sa fin encadre mon propos :
cette lignée encore fière
qui croit au livre
et qui s'évertue à écrire
ce à quoi elle tient
rien de plus rien de moins
que le passage du temps
que la vie, cette énigme ${ }^{1}$.

Soyons ce sang qui ne saurait mentir

Aborder la poésie de Sylvestre Clancier a quelque chose d'inquiétant. Le poète se dresse avec l'aura de ses multiples fonctions dans les sociétés littéraires et la présence d'un père qui nous est toujours sympathique et dont nous relisons l'œuvre avec émotion. Je ne peux pas commencer mon propos sans un envoi amical à celui qui est aussi notre père en poésie.

Je parlerai du poète en évoquant ces deux pôles, celui de l'héritage et celui de l'ouverture au monde social. Avoir pour père un homme de lettres qui a su donner une enfance heureuse à son fils, et s'attaquer à l'amélioration des soucis matériels des écrivains nous éclaire sur le poète profond qui nous fait l'honneur d'être des nôtres aujourd'hui.

J'ai cependant d'abord senti dans l'œuvre cette inquiétude que tout homme ressent devant la vie, son devenir, ses mystères. C'est cette inquiétude qui a poussé l'homme à écrire, mais d'abord à lire, à chercher

1 Sylvestre Clancier, poème inédit, Poésie sur Seine, n 54, septembre 2005, p. 7. 
chez les autres poètes des réponses à ses propres interrogations. Mais devenant ensuite plus assuré, le poète se rend parmi les hommes, leur tend la main et découvre grâce à eux la profondeur de son être, semblable aux autres hommes.

\section{L'inquiétude}

L'enfance est, pour beaucoup, un paradis dont se sent exilé l'adulte, vite poussé à faire usage de sa raison. C'est cette impression qui domine quand le poète parle de sa propre enfance, notamment dans le recueil intitulé $E_{n f r a n c e^{2}}$. Le titre joue sur les mots pour mieux associer un temps, l'enfance, et un espace, le Limousin. Toutes les notices bio-bibliographies de Sylvestre Clancier débutent par cette marque, il y ajoute même le mot "berceau " pour mieux préciser que cette enfance est le lieu d'une famille entière.

Nous savons les liens très forts qu'il entretient avec son père, particulièrement avec ce que décrit le romancier dans Le Pain noir. Le Limousin figure ainsi comme un espace-temps exemplaire, lieu d'une réalité sociale parfois dramatique, lieu des grèves des porcelainiers, lieu d'exode rural et enfin lieu de guerre avec l'épisode particulièrement tragique d'Oradour sur Glane. Ce recueil, Enfrance, s'organise autour de quatre noms de ville ou de villages : Ainé-le-château, Chézal-Benoît, Limoges et Ré la blanche qui donnent au recueil un parfum d'exotisme intérieur, rappelant un célèbre poème d'Aragon ${ }^{3}$. À côté des noms propres, un cinquième nom, commun celui-là, est rejeté à la fin pour se charger de symbole : "L'île perdue ". L'on sait donc, à la lecture de la table des matières, que l'enfance s'exile dans la mémoire.

Ainsi, outre un sentiment de bonheur qui se dégage de ce livre, nous y voyons poindre de façon récurrente un sentiment d'inquiétude. L'enfant éprouve-t-il un sentiment d'abandon quand il est saisi par la vue d'un singe de cirque épouillant son petit? L'effet de miroir est immédiat : "Comme il doit être bien dans ces bras-là ", soupire le poète qui se souvient de l'enfant fasciné par cette scène $e^{4}$. La chute de ce poème vient retentir dans la conscience d'un passé perdu, le regret d'une tendresse passée.

2 Enfrance, Proverbe, 1994.

3 Louis Aragon, "Le Conscrit des cent villages ", La Diane française, Seghers 1963 , p. 41.

4 Enfrance, op. cit., p. 19. 
Mais, paradoxalement, ce bonheur d'enfance devient, pour l'adulte, comme une charge :

\author{
Dieu avait à peine bougé \\ Bel époux de la vigne \\ Il prédit l'espoir \\ À Caïn l'enfant \\ La vie \\ Les blessures \\ La dette de l'aîné \\ Et la vue qui le rendrait fragile.
}

Tout est bien

Je ne crains pas les maux

Ma naissance est ma croix 5 .

Le monde est recréé dans avec son lot de malheurs. La vision christique finale a de quoi étonner : elle fait de l'adulte un homme en perpétuelle quête du paradis perdu, quête dont le succès lui permettrait d'alléger son faix. Espoir impossible.

Ce qui frappe surtout, dans les vers qui relatent l'enfance, ce sont les évocations des fous. On sait que la mère du poète, Anne Clancier, est psychanalyste; un passage du roman de Georges-Emmanuel Clancier, L'Eternité plus un jour ${ }^{6}$, qui vient d'être réédité, nous présente un personnage fréquentant de façon professionnelle les asiles d'aliénés. L'enfant se sent gêné par le regard de : "Tous ces fous qui te guettent »" se souvient-il. L'adulte se rappelle aussi de leur sourire. Rien d'agressif, mais un sentiment d'étrangeté, malgré une apparente sérénité qui va orienter l'enfant vers l'exploration intérieure :

\author{
$\mathrm{Tu}$ as deux ans tu es blond \\ Insouciant et rieur \\ Ta mère te filme \\ Toi qui vas te noyer ${ }^{8}$.
}

5 Une Couleur dans la nuit, Phi, coll. Graphiti, Écrits des Forges, Québec, 2004., p. 130.

6 Georges-Emmanuel Clancier, L'Éternité plus un jour, Laffont, 1969, 2005.

7 Enfrance, op. cit., p. 15.

8 Enfrance, op. cit., p. 11.

Fournier 
La distance émise par la description rapide est quasi à l'image de cette caméra qui vient mettre en évidence le désarroi. Les effets de réel accentués par le blanc entre les mots " deux ans " et " blond ", la modernité du mot " filme ", précisent une scène dont on ne pressent pas la chute. Véritable chute en effet que ce passage du présent historique au futur immédiat qui mime la descente vers l'eau. L'homme n'est pas tranquille avec lui-même. Plus loin le poète prend conscience de son reflet : " nous reconnaissons notre image trouble et déformée "? " L'âme est complexe ", nous dit-il dans L'Ame alchimiste ${ }^{10}$. Le miroir de l'eau lui révèle l'«énigmel Que chaque homme est à lui-même $»^{11}$. D'où une inquiétude intérieure. Il se croyait inentamable à son identité, une fois pour toutes. Elle se désintègre.

Le malaise étrange que le poète ressent le fait remonter bien loin dans le passé, bien plus loin que dans le simple temps de son enfance. Ou alors il s'agit de l'enfance de l'homme, celle de l'humanité. C'est ainsi qu'on remonte au premier meurtre, celui d'Abel. Un poème évoque cette descendance coupable : Hénoch, fils de Caïn, et Tubalcaïn, dont le nom est un écho à son ancêtre, le père des forgerons, c'est-à-dire le symbole du feu mais aussi celui de la construction, de l'architecture :

\author{
Si je voulais porter ma croix j'irais à pied \\ Mais j'ai préféré tuer \\ Créer Hénoch et Tubalcaïn \\ Et l'acacia refleurira ${ }^{12}$.
}

Avant l'espoir des derniers vers, le poète nous avoue son crime. Si le meurtre s'accompagne de la création, c'est qu'il y a entre les deux une nécessité. Le poète refuse l'image du Christ et de sa Passion, avec l'insistance que donne l'alexandrin, pour se tourner vers une invention porteuse d'espoir. Pourtant, ces vers proviennent d'une partie intitulée "Ecce homo ", c'est-à-dire du Christ, au début de sa Passion, couronné d'épines et présenté au peuple par Ponce Pilate. Aux yeux de Sylvestre Clancier l'homme est vu comme un Christ chargé du poids des fautes de ses ancêtres, voire de ses contemporains. L'empreinte judéo-chrétienne pèse encore sur nos consciences : comment se sentir serein devant le malheur des hommes?

9 Enfrance, op. cit., p. 40.

10 L'Âme alchimiste, Proverbe, 2003, p. 15.

11 Une Couleur dans la Nuit, op. cit., p. 22

12 Une Couleur dans la nuit, op. cit., p. 131. 
Cette mauvaise conscience débute avec le sentiment que l'adulte n'a pas su réaliser les rêves de l'enfant qu'il fut :

Peu de mots et du sens

Serait-ce un jour possible

Qu'une voix s'élève palpitante et sonore

Qu'elle célèbre la beauté de ce monde

Les enfances innocentes et les blés de l'été

Le flux et le reflux de lamer océane

Le soleil qui soudain parait au fond du pré

Dissipant la rosée et les peurs de la nuit

Que le chant du poète ravive l'espérance

Qu'il anime la flamme d'une onde plus humain?

La femme serait l'aimée et l'homme son allié

L'enfance serait reine et ses rêves adviendraient.

Que n'as-tu essayé pour qu'il en soit ainsi' ${ }^{13}$ ?

La culpabilisation sonne comme un coup de boutoir après l'idéal qu'évoque le poème, dans lequel le lyrisme se construit essentiellement d'alexandrins. Plus loin dans le même recueil, Une Couleur dans la nuit, nous trouvons l'aveu lourd malgré la légèreté de ton :

Tu t'éveillais jadis écureuil aux aguets

Espiègle émerveillé des bruits si familiers

De ta maison natale. Où est cette allégresse

Ce sentiment d'éternité toujours renouvelé?

Aujourd'hui tu t'habilles de la couleur du temps

Tu en uses comme d'un paravent

Tu fuis le vent des sentiments

Tu voulais vivre dans ta maison de Ré

$\mathrm{Tu}$ as vieilli sur le continent ${ }^{14}$.

De nombreux vers, poussés par le rythme et les rimes, soulignent une certaine nostalgie d'un bonheur passé. La seconde partie, en vers plus brefs, soutenus par la rime en [an], donne un écho significatif de la conscience coupable de l'adulte.

13 Une Couleur dans la nuit, op. cit., p. 55.

14 Une Couleur dans la nuit, op. cit., p. 63. 
Car aucune faute ne peut être rachetée. L'homme se range au rang de l'animal sacrifié en bouc émissaire :

On me sacrifie

Je crois être la voix

On me fend la croupe

Je crois être l'issue

On me brûle

Je crois vivre un songe

Je dois tendre mon âme à l'aile du vent.

Ma torture au nom de la rose

Ma croix sortie de terre

Ma passion humble mon malheur

Ma croix.

Je vois pleurer ceux qui me restent

Que leur importe mon malheur

Je suis la bête ${ }^{15}$.

Toute victime expiatoire paie une faute qui ne la concerne pas. La société humaine organise sa bonne conscience au prix de la souffrance d'un autre règne. L'alternance des " je " et des " on ", l'insistance de la violence physique avec les mots "fendre ", "brûler ", " torture ", le rappel de l'aventure christique " ma croix ", tous ces indices renvoient le poète au cri de la bête que le poète ne peut pas même proférer. La brièveté des vers, la multiplicité des actions et l'insistance de la première personne viennent renforcer la chute, "Je suis la bête ". Le poète dénonce violence, à travers la religion, ou plutôt ses rites au service du mal, qu'il faut bien justifier parce qu'il existe, et qu'il est difficilement supportable en toute bonne conscience.

L'inquiétude du poète est profonde et c'est ce qui motive son écriture qui devient le seul lieu par où l'homme peut espérer croire en une certaine rédemption, si nous poursuivons la métaphore christique, suggérée par l'auteur lui-même, ou, pour le moins, un certain apaisement, voire une catharsis.

15 Une Couleur dans la nuit, op. cit., p. 132. 


\section{II. Écriture}

L'écriture offre d'emblée l'usage de la métaphore qui va calmer cette inquiétude en l'exprimant. Elle permet de fonder un imaginaire dans lequel l'esprit prend le champ nécessaire pour mieux se retrouver. L'écriture s'attaque d'abord au temps puis se révèle à elle-même en tant que matière, et la lecture des aînés ouvre des voies fascinantes. L'écriture est une quête.

La première tâche de la poésie est d'aborder la fuite du temps, une certaine vision du futur. Un poème de L'Herbier en feu témoigne de ce sentiment dans un raccourci surprenant : " [...] des poissons filants des étoiles sans branche/ écaillent notre destinée ${ }^{16}$. Le jeu de mots entre les poissons et les étoiles relie l'eau et le ciel. L'un comme l'autre représente ce miroir placé en face de nous. Le ciel est près de se dissiper dans nos rêves. Notre destin persistera-t-il à se définir dans l'élément plus prosaïque de l'eau terrestre? Le cours de l'eau insiste sur le passage du temps alors que les étoiles nous font rêver d'une illusoire éternité. Penchons-nous alors vers ce qui nous est promesse d'espoir.

Trésors de la vie dérobés
par d'imbéciles guerriers
aux doigts vils
à l'ardeur des vautours
Un jour on reverra ces ors
filles, écoliers, voyelles
vignes rousses, azurs d'été
grande beauté des prés
magnifiés par l'alphabet
la passion des conteurs ${ }^{17}$.

Cet espoir est nourri du souvenir des histoires narrées durant l'enfance. L'écriture vient dépasser les aberrations de la guerre. On sera sensible aux jeux phoniques qui rassemblent les éléments du poème : "un jour on reverra ces ors [...] vignes rousses, azurs d'été », allitération en [R], « filles, écoliers, voyelles " allitération en [L], assonance en [i], et la caisse de résonance que constituent les multiples emplois de la lettre " 0 " : dans " jour ", " on ", " ors ". Ce maillage phonique densifie le poème à la façon

16 L'Herbier en feu, op. cit., p. 22.

17 Présent Composé, Écrits des Forges/Proverbe, 1996, p. 39. 
d'un réseau rassurant et révèle la foi de l'auteur pour le bel ouvrage, celui qui permet le creusement de soi par le vers. Les coupes rythmiques soulignent à chaque fois un sens précis tandis que les rimes concourent à une certaine harmonie. "Un jour " le poète trouvera son propre langage pour fonder un nouvel avenir n'ayant plus à porter la croix du passé des hommes.

L'art poétique de Sylvestre Clancier se construit sur la page : il laisse l'espace du blanc donner au vers toute son autonomie et sa force de sens. Cet espace parfois s'insère à l'intérieur du vers, écartant alors un mot du groupe pour lui donner plus de poids, ce qui fait penser à André du Bouchet. Ce blanc interstitiel vient combler l'absence de ponctuation : un poème pour être vu et pour faire savoir comment il doit être dit. À cet égard, on pense à Jean Follain dont Sylvestre Clancier a suivi la leçon de la modestie et de liquidité de la phrase dans une expression qui amène, paradoxalement, au mystère.

Ainsi Sylvestre Clancier dessine-t-il autour de lui un cercle des poètes parmi ceux dont la voix et la vie, les deux mots rassemblés dans le mot " voie ", se retrouvent dans le titre La Voie des poètes ${ }^{18}$. Cet essai remarquable et original fait la part belle à ceux qui ont vécu une vie qui ressemblait à leur poésie. Nous nous attarderons sur trois noms : Daumal, Miron et Nerval, qui se retrouvent dans sa poésie.

Couleur dans la nuit commence par ce poème dédié à Gaston Miron :

Il marche

Il défend sa peau

Le poète

Toute sa peau

Refuse l'inacceptable

Et la désespérance

Car il voit ce qu'il voit

Le poète

Et parie pour demain.

Debout, il s'insurge

Il a des mots cogneurs

Raboteux, ravageurs

Dont je reprends l'écho ${ }^{19}$.

18 La Voie des poètes, Les Lettres du Temps, 2002.

19 Une Couleur dans la nuit, op. cit., p. 9. 
À travers la tautologie apparente (" il voit ce qu'il voit ") qui reprend le thème de la "voyance " des initiés tels Rimbaud ou Marc Alyn (avec ses Miroirs voyants), Sylvestre Clancier rend compte de la violence efficace de l'action par les mots " cogneurs ", tel " défendre sa peau ". De L'Homme rapaille ${ }^{20}$, le poète se sent le frère parce que celui-ci est parvenu à rassembler ce qui est épars.

Daumal, de son côté, a tenté une voie mystique avec le Mont analogue ${ }^{21}$. Notre poète le révèle comme un : "Génie de la poésie et de l'exaltation/ Géomètre de nos désirs enfouis "22. Ces deux vers débutent par la lettre " G " qui pourrait signifier aussi le mot " génération ", Sylvestre Clancier bâtissant là une nouvelle généalogie. On parlera aussi de " gnose » dans la tradition cabalistique qu’apprécie Sylvestre Clancier. Par le mot " génie » le poète entend une âme nouvelle, et par "géomètre " un arpenteur de l'intérieur. Le travail du poète, ingénieur et architecte de lui-même, impose une sérieuse " ascèse mystique ".

Enfin Nerval qui ne s'intéresse que fort peu, dans son Voyage en Orient, au tombeau du Christ. Davantage l'intéressent les mythes fondateurs de l'humanité, notamment celui de Caïn, que nous venons de voir, et celui de Salomon, l'architecte du Temple de Jérusalem. Nerval, au-delà de la référence chrétienne, et comme Gaston Miron, " voit ", grâce à un troisième oil, celui-là tout intérieur. La folie de Nerval rapproche Sylvestre Clancier du souvenir de ces fous que sa mère soignait. La folie, pour qui cherche en sa plus grande intériorité, n'est-elle pas davantage un cheminement plutôt qu'une maladie mentale socialement dérangeante?

\author{
Il t'en aura fallu \\ De la belle amertume \\ Pour en venir à bout \\ De ces jours inutiles \\ De ces jours de vague à l'âme \\ De ta mélancolie de Prince d'Aquitaine \\ À la tour abolie ${ }^{23}$.
}

20 Gaston Miron, L'Homme rapaillé, Gallimard, coll. Poésie, 1998. Rapaillé : Expression québécoise : se dit d'une chose fragmentée, éparpillée, dont on rassemble les morceaux.

21 René Daumal, Le Mont analogue, 1952.

22 Une Couleur dans la nuit, op. cit., p. 15.

23 Fragment de poème inédit. 
Cette folie, Sylvestre Clancier la ressent comme une solidarité.

L'écriture seule peut permettre de ne pas s'épancher dans la folie. Dès qu'un poète entreprend un poème, il doit lutter avec l'ange du langage, dirait Marc Alyn. Cette lutte, il la mène contre plusieurs démons : celui de sa propre difficulté à trouver le mot juste, celui de la copie des vers repris aux maîtres, et enfin celui du langage de tous les jours, dont le poète doit s'affranchir en gardant des mots simples éclairés par des effets de métaphores, de rythme et de sonorités, pour trouver son propre ton. C'est ainsi qu'on en arrive au langage second, en quoi consiste la littérature et plus précisément la poésie.

La difficulté de l'écriture est rendue sensible dans un poème du Présent composé:

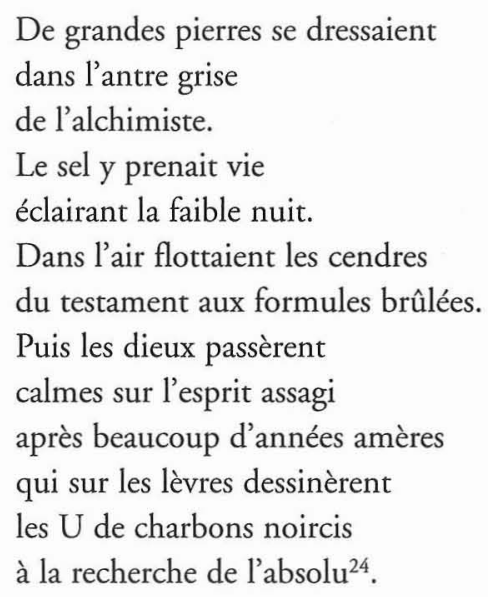

Nous retrouvons l'antre de Tubalcaïn, le maître des alchimistes. L'alchimie, celle des mots, est ici vécue, dans une atmosphère picturale à la Rembrandt, comme une quête impossible de la parole perdue.

En effet, il s'agit, nous dit Sylvestre Clancier dans l'Ame alchimiste, de trouver "une autre écriture ${ }^{25}$, de "retrouver une harmonie " dans le " testament " aux « formules brûlées ». Cet idéal désespéré consiste à aller au-devant de la parole perdue. L'infinitif "retrouver" ne sonne pas ici comme un ordre mais bien plutôt comme un souhait, pour qu'enfin jaillisse des lèvres du poète ce cri qu'un autre poème célèbre : « La langue

24 Le Présent composé, Écrits des Forges/Proverbe, 1996, p. 12.

25 L'Âme alchimiste, op. cit., p. 19. 
retrouvée enfin ${ }^{26}$, au moment " où l'oreille/ est maîtresse du son ". La musique fait partie de la création poétique, les sons émis par la langue doivent s'ordonner en harmonie; le poète est à l'écoute de cette musique intérieure qu'il essaie de transcrire pour que son âme s'élève.

À partir de cette musique, le poète se doit de trouver son propre verbe : "Chaque homme en vivant crée sa phrase [...] Telles sont les phrases du Livre/ Mortelles ${ }^{27}$. L'adjectif en rejet redoutable qualifie les phrases. $\mathrm{Ne}$ nous leurrons pas : il ne s'agit pas d'une création éternelle et définitive. Nous ne quittons pas le règne du vivant, les phrases sont éphémères. Mais l'empreinte mythique de Mallarmé demeure revendiquée avec la majuscule de "Livre ". Cependant c'est ce même caractère périssable des phrases que le poète a prononcées depuis l'origine qui l'incite à poursuivre l'œuvre commencée, ne se sentant pas le droit d'interrompre l'œuvre en devenir.

L'écriture permet au poète de prendre des distances avec son inquiétude, de se confronter aux autres poètes, de s'initier soi-même aux mystères de la création et entraîne enfin, sans dogmatisme, la communication avec les frères humains dans une voie que l'on sait gratifiante.

\section{Le Compagnon}

L'entreprise poétique devient alors un compagnonnage avec la nature, une communauté avec les poètes, une fraternité avec les hommes.

Cette fraternité s'exerce d'abord avec le monde qui l'entoure : les mondes animal et végétal, et avec celui de l'enfance.

Le monde animal tient une grande place dans l'univers poétique de Sylvestre Clancier, à tel point qu'il lui a consacré un recueil entier : $L^{\prime}$ Animal anime $e^{28}$. Ce titre est déjà tout un programme. L'animal est en soi un être vivant dont le nom vient de " anima ", "souffle de vie ", de même que le verbe "animé ". Ne voyons donc pas trop rapidement une redondance dans ce titre, mais bien plutôt un jeu de mots. C'est lui qui les anime, au double sens de donner une âme et de mettre en mouvement. Ce bestiaire n'est pas un catalogue de naturaliste mais une vision particulière que porte l'homme sur les animaux. Il s'agit d'un imaginaire dans lequel le poète retrouve des racines mythiques. Il s'adresse ainsi à un animal proprement fabuleux, Mélusine, mi-animal, mi-femme : «Tu es la voix de

26 L'Âme alchimiste, op. cit., p. 45.

27 Une Couleur dans la nuit, op. cit., p. 124.

28 L'Animal animé, Proverbe, 1999. 
l'ombre/ Notre animalité ${ }^{29}$. Il importe de souligner le lien que tisse le poète entre ceux qu'Audiberti appelait les "frères inférieurs ", et les hommes.

Donnons au passage quelques traits qu'il prête à certains animaux. Le bison est vu comme "Compagnon de l'Indien/ Âme de l'Amérique "; le chacal comme "veilleur des morts "; le Dindon, "Quichotte ou Tartatrin/ Prosaïsme ou lyrisme " et "Jonas/ l'initié ". Ce qui est remarquable dans ce bestiaire, c'est le renvoi à une valeur symbolique, le peuple amérindien pour le bison, l'Egypte pour le chacal, le mythe biblique pour Jonas et les littératures traditionnelles et populaires pour le dindon.

C'est donc par l'intermédiaire de l'imaginaire des cultures que le poète entre en contact avec les animaux. Nous assistons à une fraternisation par le bénéfice de symboles universels qui donnent aux animaux un supplément d'âme.

Le lien avec le minéral n'est pas moins fort, comme l'atteste le recueil Pierres de mémoire ${ }^{30}$.

\author{
Pierres des mythes \\ Roches fossiles qui abritez les morts \\ Ou leurs métamorphoses \\ Vous rassurez celui qui ne croit plus à rien \\ Témoignant qu'hier a bien existé avant demain ${ }^{31}$.
}

Les pierres portent le passé et assurent l'avenir; par elles, la leçon des morts se transmet. Elles nous rappellent constamment à la réalité de l'Histoire, à la force des enseignements du passé. Comme pour les animaux Sylvestre Clancier se plaît à passer en revue nombre de roches (l'or, la calcédoine, l'opale, l'hématite, le grès, le graphite, pour n'en citer que quelques-unes) pour en révéler l'âme. Le titre, Pierres de mémoire, dit assez qu'elles sont pour lui le signe d'une composante essentielle de la proximité, de l'homme avec les éléments de la nature.

Les pierres primordiales reflètent les âges de la matière; mais Sylvestre Clancier s'intéresse aussi aux pierres façonnées par les hommes, car elles participent toujours à l'accompagnement d'un sentiment du sacré ou d'un rituel :

29 L'Animal animé, op. cit., p. 45.

30 Pierres de mémoire, Écrits des Forges/Proverbe, 2000.

31 Pierres de mémoire, op. cit., p. 41. 


\title{
Pierres dressées \\ Pierres branlantes \\ Bonnes fontaines \\ Vous élevez l'esprit \\ De celles et de ceux \\ Qui vous visitent ${ }^{32}$.
}

On pense à Guillevic et au sacré sans Dieu $^{33}$ : sans doute le sentiment estil un peu différent, mais nous retrouvons cette mystique laïque qui a pour objet d'ajuster l'homme à son destin.

La solidarité avec le monde animal et minéral se complète avec le règne végétal dans L'Herbier en feu : "Il y a au loin derrière l'horizon/ des plantes grimpantes/ Elles nous attendent/ Leurs bras nous enlaceront/ Leur œil d'un bleu luisant nous reconnaîtra/ en toute occasion ${ }^{34}$. » Un anthropomorphisme semble ici se dégager plus nettement que précédemment : le végétal s'anime, dans ce poème, à la mémoire de l'amitié. Chez Sylvestre Clancier, tout est humain. On comprend mieux qu'il n'y a pas d'échelle entre les règnes du vivant et que le contact avec la différence apporte au poète une aptitude d'accueil à tout ce qui est en vie.

Mais l'attachement aux hommes est sans doute le trait le plus caractéristique de la poésie de Sylvestre Clancier, Il se veut le véritable compagnon, comme de ses frères inférieurs, de ses frères humains. Chacun représente un avatar de la totalité.

\author{
Amie écoute ma peine \\ mon souffle retenu \\ Un fou sans vie des rêves maintenus \\ Des riens \\ Le seuil franchi \\ Le faux herbier du temps \\ La lumière qui retombe \\ Les gens que l'on ramasse \\ Un homme en pleurs à sa fenêtre \\ caresse le blé qui danse \\ $\mathrm{Au}$ fond des tombes quelques vers luisants
}

32 Pierres de mémoire, op. cit., p. 39.

33 Jacques Lardoux, Le Sacré dans Dieu dans la poésie contemporaine, Octavio Paz, Guillevic, Gottfried Benn, Yves Bonnefoy, éd. Prométhée, 1993.

34 L'Herbier en feu, op. cit., p. 22. 
De l'ombre dans le sang si l'on va plus avant

Un masque où blanchit le carnaval du temps

En vitrine de larges épées pour la fête

Un nouveau-né qui détourne la tête

Et le gui et le houx qu'on laisse suspendus.

Unis en un seul être nous étions forts

et plein d'espoirs ${ }^{35}$.

Dans ce très beau poème, je souligne ces vers : « La lumière qui tombe/ Les gens que l'on ramasse ", dans lesquels l'auteur par une antithèse des plus simples identifie le temps, la lumière, la détresse et la solidarité. Le poète remarque les moindres signes des indices du malheur. Une forte tristesse vibre dans ses pages où l'échec s'inscrit dans le titre. L'Herbier en feu, est donc un "faux herbier du temps": le temps ne recueille jamais rien et surtout pas un souvenir en forme de pétale séché, peut-être demeure-t-il seulement le livre. Inquiétude qui ne saurait se passer d'un compagnonnage : "Amie écoute ma peine ". C'est ainsi que dans le même élan où il convoque l'autre à l'écouter, il se lance au-devant de l'autre par solidarité. Il se dit lui-même " compagnon des autres, des anciens/ les morts $~^{36}$; c'est-à-dire qu'il se sent le devoir de dresser une ligne de vie entre les morts et les vivants. Sylvestre Clancier est un passeur, le mot est à la mode, je le reprends pourtant, pour rendre hommage à celui qui fait se reconnaître les poètes entre eux, jeunes et moins jeunes, sans oublier de convoquer tous ceux qui les ont nourris dans le passé.

Ce compagnonnage s'appuie sur un rituel. Le monde poétique ne doit pas se fermer sur lui-même mais se faire connaitre comme exemplaire. $\grave{A}$ travers les poètes, Sylvestre Clancier souhaite que toute assemblée d'hommes soit une réunion de frères, tous "lointains cousins ", qui lui offrent une découverte de lui-même. Cela ne va pas sans une initiation, étape qu'on retrouve dans toutes les sociétés, parcours à la fois individuel et collectif, démarche d'humaniste.

Une lettre, un signe, un pas.

Il est déjà à la porte du temps

pour continuer la marche,

pousser les vantaux,

avancer dans la nuit,

35 L'Herbier en feu, op. cit., p. 20.

36 L'Herbier en feu, op. cit., p.

\section{LittéRéalité}


sous le regard protecteur de ceux qui l'ont précédé.

Ils sont là près de lui,

ils l'accompagnent,

soutiennent son effort.

Les vanités, les faux-semblants oubliés,

calme et déterminé,

il va goûter la nuit.

L’or véritable qui est celé en lui

a l'éclat d'une fleur ${ }^{37}$.

Ces hommes lui sont nécessaires dans sa démarche ésotérique qui le mène dans la nuit où pourtant fleurit le minerai. Il faut insister sur cette initiation, descente intérieure, grâce à " une étrange alchimie ". La compagnie des autres n'est là que pour soutenir une solitude essentielle. L'esprit du rituel aide l'homme à se trouver lui-même. Le risque d'une défaite, d'un désespoir que pourrait apporter la descente dans les gouffres du moi, la crainte de la folie que pourrait amener la confrontation avec soimême, tous ces dangers sont écartés par la présence d'une communauté. Mais il ne faut pas croire que le cheminement soit pour autant aisé :

Ainsi devrons-nous longtemps cheminer

Avant de gravir cette marche dernière

Avant d'atteindre cette porte secrète

Lâme légère, l'alphabet retrouvée ${ }^{38}$.

Sylvestre Clancier est intéressé par la démarche cabalistique qui fait le lien avec le langage, ses ramifications avec les passés obscurs, avec des secrets intimes. Observons que cette progression se fait dans le sens de la montée. Il n'y a pas loin de cette imaginaire union d'âmes à une élévation mystique :

Bonheur du voyage entrepris

qui s'est prolongé dans la nuit,

nous avons été portés

élevés. ${ }^{39}$

37 Une Couleur dans la nuit, op.cit., p. 91.

38 Une Couleur dans la nuit, op.cit., p. 94.

39 Une Couleur dans la nuit, op. cit. 
Le poète est un voyageur que l'expérience élève. Sans donner au mot transfigurer une acception immodeste et démesurée, l'on doit pourtant tenir compte de l'action du parcours poétique sur le sujet. Dans la continuité d'une quête prégnante tout au long de son écriture l'opération poétique n'est-elle pas chez Sylvestre Clancier, la réalisation, indéfiniment à reproduire, de l'idée d'un travail de transsubstantiation?

\title{
Conclusion
}

Sylvestre Clancier est bien plus qu'un poète, c'est un homme qui partage, qui s'offre, par l'écriture mais aussi par la vie sociale, aux autres, pour aimer, soutenir, autant lui-même que les autres, les autres à travers lui-même.

\author{
Si les jeux n'étaient plus des jeux \\ et la poussière un nuage \\ si les ombres étaient la lumière \\ un profil un visage \\ si les rêves étaient faits divers \\ et la veille le sommeil \\ les mots sonneraient plus vrais \\ ils nommeraient les plus simples choses \\ le réel serait un poème \\ et la poésie la vie ${ }^{40}$.
}

Ce poème tiré de Une Couleur dans la nuit se veut un art poétique construit autour d'un fort parallélisme. On demeure au mode conditionnel pour affirmer une foi dans le langage seul capable, mais seulement dans sa partie la plus âpre et la plus difficile, la poésie, d'affronter la vie. C'est ainsi que l'inquiétude à partir de laquelle le poète éprouve la nécessité de l'écriture transforme celle-ci en ouverture. L'homme seul et inquiet est devenu le compagnon des hommes. 